\title{
"Asthma": receta de tratamiento en el Virreinato de la Nueva Granada en el siglo XVIII
}

\author{
"Asthma": Prescription for treatment in the \\ Viceroyalty of New Granada in the 18th century
}

\author{
Eric Yesid Canaria Ascencio, MD¹ Alberto Gómez Gutiérrez, PhD² Jorge Uribe Vergara, PhDc ${ }^{3}$ \\ Julio César Martínez Lozano, MD, MSc ${ }^{4}$ Ignacio Briceño Balcázar, MD, PhD. ${ }^{4}$
}

\section{Resumen}

OBJETIVO: presentar una receta médica del siglo XVIII en el Virreinato de la Nueva Granada, que describe el tratamiento de asma.

MATERIALES Y MÉTODOS: búsqueda en el archivo histórico de la Biblioteca Octavio Arizmendi Posada en la Universidad de La Sabana (Chía, Colombia), donde se conserva una colección de un centenar de recetas médicas coloniales del siglo XVIII; se realiza una transcripción de una de ellas, titulada "Asthma", con una ulterior revisión de la literatura científica pertinente.

CONCLUSIONES: esta receta médica colonial muestra su utilidad para calmar los síntomas por asma y constituye un claro ejemplo de terapias comparadas a través del tiempo. Además, esta prescripción constituye un ejemplo documental de la evolución histórica de la medicina, desde los tiempos coloniales de los entonces territorios de ultramar de la Corona Española.

Palabras clave: historia de la medicina, Colombia, siglo XVIII, asma, prescripciones.

\section{Abstract}

OBJECTIVE: To present a medical prescription of the eighteenth century in the Viceroyalty of New Granada, which describes the treatment of asthma.

MATERIALS AND METHODS: A documentary search in the historical archive of the Octavio Arizmendi Posada Library at the University of La Sabana (Chía, Colombia) was performed, where a collection of a hundred colonial medical recipes of the $18^{\text {th }}$ century is preserved; we present a transcription of one of them, entitled "Asthma", with a subsequent revision of the pertinent scientific literature.

CONCLUSIONS: This colonial prescription shows its usefulness for treating symptoms induced by asthma and constitutes a clear example of compared therapeutics through the ages. In addition, this prescription should also be recognized as a documentary example of the historical evolution of medicine, since the colonial times of the overseas territories of the Spanish Crown.

Keywords: History of medicine, Colombia, 18th century, asthma, prescriptions.

${ }^{1}$ Facultad de Medicina, Universidad de La Sabana. Bogotá D. C., Colombia.

${ }^{2}$ Biólogo, Instituto de Genética Humana, Facultad de Medicina, Pontificia Universidad Javeriana. Bogotá D. C., Colombia.

${ }^{3}$ Historiador, Candidato a Doctor en Ciencias Sociales, Pontificia Universidad Javeriana. Bogotá D. C., Colombia.

${ }^{4}$ Biociencias, Facultad de Medicina, Universidad de La Sabana. Bogotá D. C., Colombia.

Recibido: 25/7/2018. Aceptado: 30/10/2018. 


\section{Introducción}

El archivo histórico de la biblioteca Octavio Arizmendi Posada en la Universidad de la Sabana (Chía, Colombia) cuenta con una colección de un centenar de recetas médicas coloniales del siglo XVIII, donadas por el Padre Cipriano Rodríguez Santa María, epónimo institucional del archivo (1).

El origen etimológico del término para esta condición proviene del griego $\tilde{\alpha} \sigma \theta \mu \alpha$ (ásthma) que literalmente significa "jadeo" o "sofoco", uso registrado por Homero (siglo VIII a. C.) por primera vez en la Ilíada como un padecimiento manifestado por Héctor y por Áyax, héroes troyano y aqueo respectivamente, durante las numerosas lides que enfrentaron ambos grupos. Asimismo, la enfermedad se describió previamente en el papiro de Ebers, un tratado médico del antiguo Egipto $(2,3)$.

El asma es una enfermedad heterogénea, generalmente caracterizada por una inflamación crónica de las vías respiratorias. Por consenso universal, se define como la historia de síntomas respiratorios (sibilancias, dificultad para respirar, opresión en el pecho y tos) que varían con el tiempo y en intensidad, junto con la limitación variable del flujo de aire espiratorio $(4,5)$.

Estas variaciones de los síntomas a menudo se desencadenan por diversos factores como el ejercicio, la exposición a alérgenos o irritantes, cambios en el clima $\mathrm{o}$ infecciones virales respiratorias. En cuanto a la epidemiología, afecta al 1\%-18 \% de la población en diferentes países, un rango amplio que depende de las particularidades de cada territorio. Como punto importante, es de aclarar una disminución de la tasa de hospitalizaciones y muertes por asma en muchos países, aunque se debe resaltar la cuantiosa carga que el asma representa para los sistemas de salud en numerosos estados (4).

Actualmente se dispone de un manuscrito original de una receta médica del siglo XVIII para tratar el asma, que se puede observar en las Figuras 1 y 2 (1), la cual está basada en una publicación original de 1757 (6).

La relevancia del presente artículo reside en que explora y fundamenta el entendimiento actual de algunas recomendaciones médicas utilizadas en la historia de la medicina. El objetivo principal de nuestro trabajo es presentar el contexto de formulación de una receta médica del siglo XVIII en el Virreinato de la Nueva Granada (actual Colombia), la cual contiene recomendaciones para el tratamiento del asma, y también analizar si estas son útiles para el mejoramiento de esos síntomas descritos.

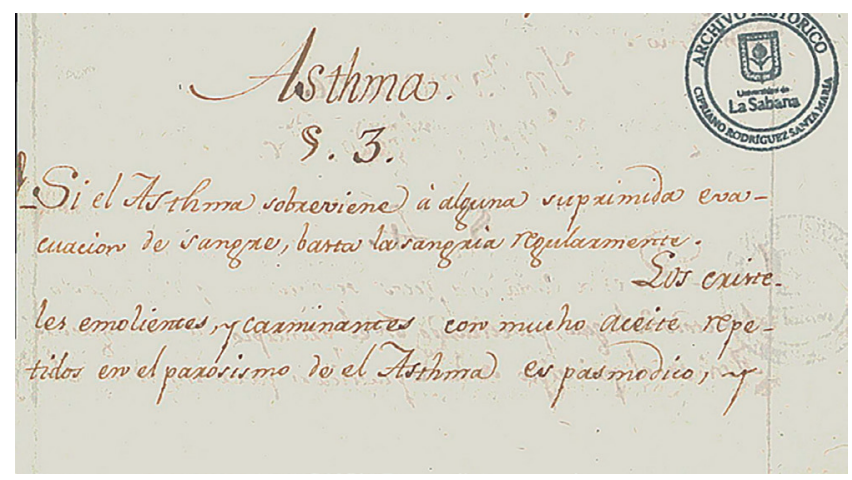

Figura 1. Receta médica titulada "Asthma". Tomada de: Archivo Histórico Cipriano Rodríguez Santa María (Universidad de La Sabana), Fondo Manuel María Mosquera, Caja 10, Carpeta 2, folios 98, recto 98, verso.

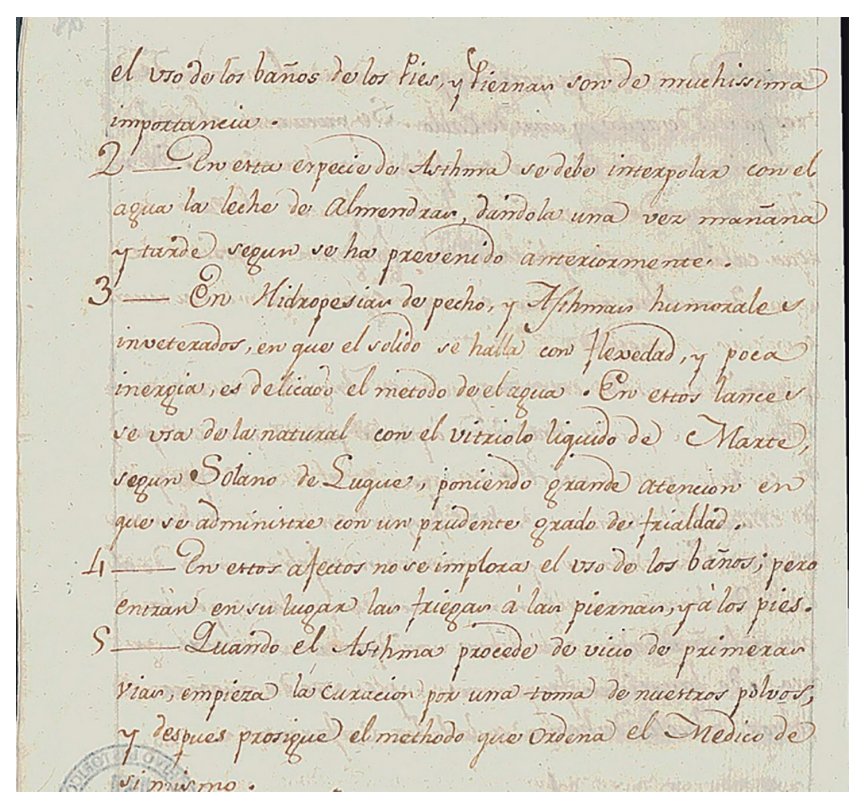

Figura 2. Receta médica titulada "Asthma". Tomada de: Archivo Histórico Cipriano Rodríguez Santa María (Universidad de La Sabana), Fondo Manuel María Mosquera, Caja 10, Carpeta 2, folios 98, recto 98, verso. 


\section{Materiales y métodos}

Se procedió a realizar una búsqueda documental en el archivo histórico de la Biblioteca Octavio Arizmendi Posada en la Universidad de La Sabana, en la que se encuentra la receta "Asthma"; ulteriormente, se realizó una revisión de la literatura disponible sobre los elementos a los que hace referencia la receta en bases de datos.

\section{Análisis del documento fuente}

En el documento fuente, que se transcribe a continuación, se observa una secuencia de recomendaciones diversas para el asma.

\section{Asthma}

1. Si el Asthma sobreviene à alguna suprimida evacuación de sangre, basta la sangría regularmente. Los cristeles emolientes, y carminantes con mucho aceite repetidos en el parosismo de el Asthma espasmodico, y el uso de los baños de los pies, y piernas son de muchísima importancia.

2. En esta especie de Asthma se debe interpolar con el agua la leche de almendras, dándola una vez mañana y tarde según se ha prevenido anteriormente.

3. En Hidropesías de pecho, y Asthmas humorales inveterados, en que el solido se halla con flexedad, y poca inergia, es delicado el método de el agua. En estos lances se usa de la natural con el vitriolo liquido de Marte, según Solano de Luque, poniendo grande atención en que se administre con un prudente grado de frialdad.

4. En estos afectos no se implora el uso de los baños; pero entran en su lugar las friegas à las piernas, $y$ à los pies.

5. Quando el Asthma procede de vicio de primeras vias, empieza la curación por una toma de nuestros polvos, y despues prosigue el método que ordena el Medico de si mismo.

De acuerdo con el punto 1, si la patología era posterior a una pérdida de sangre, bastaba usar la sangría como una cuasipanacea, tal y como se utilizaba para numerosos males en una práctica que perduró por si- glos. Está presente el uso de "cristeles", los cuales son un sinónimo de enemas, pero no se especifica claramente su uso. En el caso de paroxismos por asma, se resalta la importancia de los baños en los miembros inferiores, como uso de la hidroterapia.

La hidroterapia se define como el uso externo o interno del agua en cualquiera de sus formas (agua, hielo, vapor) para la promoción de la salud o el tratamiento de diversas enfermedades con diferentes temperaturas, presión, duración y sitio de aplicación, de acuerdo con sus potenciales efectos benéficos en el organismo $(7,8)$.

No se especifica claramente la temperatura del agua, por lo que es posible que se tratara de baños simples con agua fría (9). Esto se fundamenta en que los procedimientos locales de frío ayudan a mejorar la permeabilidad bronquial, y la exposición al calor provoca su empeoramiento (7). En el ejercicio basado en el agua para tratar el asma, falta más información para considerar eventuales resultados positivos (10).

En el punto 2 se describe la interpolación con leche de almendras, un sucedáneo de la leche de vaca sin contenido de lactosa y con ausencia de lipoproteínas de baja densidad. Aparte de su valor nutricional y sus características descritas actualmente (11), no es clara su utilidad en esta patología.

En el punto 3 se usa la expresión "hidropesía de pecho", que corresponde a una retención de líquido en el tórax, sin especificar una categoría de gravedad, como una característica semiológica cuyo rango abarca desde la tos con secreción hasta un franco edema pulmonar. Por ende, por obvias razones se proscribe la hidroterapia, usando en su lugar el vitriolo líquido de Marte, correspondiente a sulfato de hierro, enfatizando en el grado de frialdad del solvente. Este producto ya era incluido en las boticas neogranadinas coloniales y contaba con otras indicaciones $(12,13)$.

En el punto 4, se reitera el uso de la hidroterapia en miembros inferiores, sin baños, pero sí con la fricción física del agua.

En el punto 5, se recomienda usar "nuestros polvos" sin mayores datos sobre los componentes de estos. 
Se observa así que, a la luz de los conocimientos actuales, la mayoría de esta serie de recomendaciones era útil para tratar el asma, contribuyendo a una mejoría sintomática. Debe considerarse que estas disposiciones eran reguladas por la institución del protomedicato, de origen peninsular, pero eventualmente adaptadas a la situación local de aquel tiempo, como lo sugiere el uso de "nuestros polvos" (14).

En contraposición al texto precedente, el tratamiento actual del asma cuenta con 2 objetivos a largo plazo del manejo de esta patología: el primero, lograr un buen control de los síntomas y mantener unos niveles de actividad normales; y el segundo, minimizar el riesgo futuro de exacerbaciones, la limitación del flujo de aire y los efectos secundarios asociados. En consecuencia, la base del tratamiento consiste en la prescripción de medicamentos controladores (que reducen la inflamación de las vías respiratorias, controlan los síntomas y reducen los riesgos futuros, como las exacerbaciones y la disminución de la función pulmonar) y de medicamentos de rescate que se brindan a todos los pacientes para aliviar (según sea necesario) los síntomas intercurrentes, incluso durante la agravación del asma o sus exacerbaciones. Esto se logra con una metodología estandarizada paso a paso, intensificándola o reduciéndola de acuerdo con la respuesta terapéutica (4).

\section{Conclusiones}

Con base en las recomendaciones transcritas y descritas, se puede postular que las indicaciones registradas en el manuscrito colonial neogranadino podrían corresponder con los medicamentos actuales de rescate para el asma, caracterizándose su utilidad en el manejo de los síntomas respiratorios por asma, sin controlar la patología de forma definitiva.

Con el presente análisis, se evidencia el loable esfuerzo de los facultativos coloniales del Virreinato de la Nueva Granada (actual Colombia) en el empleo de recetas como la que se describe aquí para calmar los síntomas respiratorios del asma. Este ha sido un padecimiento incapacitante, adverso y potencialmente mortal para la humanidad desde los tiempos en los que el conocimiento médico y científico estaba apenas en desarrollo para los estándares modernos.
Finalmente, debe destacarse el esfuerzo para la conservación de estos documentos por parte del archivo histórico de la Biblioteca Octavio Arizmendi Posada de la Universidad de La Sabana. Estos documentos tienen un indudable y valioso valor histórico, y su consulta permite recrear en buena parte la evolución de la medicina del siglo XVIII al siglo XIX en el entonces Virreinato de la Nueva Granada.

\section{Conflicto de interés}

No se indican conflictos de interés por parte de los autores.

\section{Agradecimientos}

Los autores reconocen la generosa asistencia ofrecida por Marcela Revollo Rueda, directora de los Archivos Históricos de la Biblioteca Octavio Arizmendi Posada en la Universidad de La Sabana.

\section{Referencias}

6. Universidad de la Sabana. Archivo Histórico Cipriano Rodríguez Santa María - Fondo Manuel María Mosquera-Caja 10 - Carpeta 2 folios 98 recto 98 verso.

7. Gurrola Silva A, Huerta López JG. Historia del asma. Alergia, Asma e Inmunol Pediátricas. 2013;22(2):77-86.

8. Murtagh P. Asma: ayer y hoy. Arch Argent Pediatr. 2009;107(2):146-51.

9. Global Initiative For Asthma G. Global Strategy for Asthma Management and Prevention. Global Initiative for Asthma; 2018.

10. Navarrete Rodríguez E, Sienra Monge JJL, Pozo Beltrán CF. Asma en pediatría. Rev la Fac Med la UNAM. 2016;59(4):5-15.

11. Carvallo de Castro JI. La Verdad Desnuda: Arcanidades De El Medico De Sì Mismo, Descubiertas À La Luz De El Desengaño. Biblioteca Nacional [Internet]. 1757 [acceso el 12 de julio de 2018]. Disponible en: http://bdh-rd.bne.es/viewer. vm?id $=0000079809$ \&page $=1$.

12. Mooventhan A, Nivethitha L. Scientific evidence-based effects of hydrotherapy on various systems of the body. N Am J Med Sci. 2014;6(5):199-209.

13. Saz P, Ortiz M. Hidroterapia Agua y enfermedad. Farm Prof. 2005;19(4):84-8.

14. Pazos Rosales JM, González A. Técnicas de hidroterapia. Hidrocinesiterapia. Fisioterapia. 2002;24(monográfico 2):34-42.

15. Grande AJ, Silva V, Andriolo BN, Riera R, Parra SA, Peccin MS. Water-based exercise for adults with asthma. Cochrane Database Syst Rev. 2014;(7):CD010456. 
16. García-Saavedra NM. Bebidas Vegetales. Universidad Complutense [Internet] 2017 [acceso el 14 de julio de 2018]. Disponible en: http://147.96.70.122/Web/TFG/TFG/Memoria/ NATALIA MORALEJA GARCIA-SAAVEDRA.pdf.

17. Vásquez Carvajal C, Uribe Vergara J, Martínez Lozano JC, Gómez Gutiérrez A, Briceño Balcázar I. Opilación y supresión de menstruos: una receta médica del siglo XVIII en el Nuevo Reino De Granada. Rev Colomb Obstet Ginecol. 2018;69(1):65-70.
18. Pico RP. Las boticas en el Nuevo Reino De Granada a finales del período colonial: el lento camino hacia la modernidad. Rev Med. 2015;37(3):223-41.

19. Gardeta Sabater P. El Real Tribunal del Protomedicato en la Audiencia de Santa Fe durante la segunda mitad del siglo XVIII. Un acercamiento al estudio de las transformaciones de esta institución española. Dynamis Acta Hisp ad Med Sci Hist Illus. 1992;12(1):209-24. 